\title{
Bias in Artificial Intelligence and Machine Learning
}

\author{
Raghavi Dube and Jeenath Shafana N \\ Department of Computer Science, SRM Institute of Science and \\ Technology, Ramapuram Chennai, Tamil Nadu, India \\ Corresponding author email: raghavi.03@rediffmail.com
}

\section{ABSTRACT}

Artificial Intelligence (AI)-based systems are firmly ingrained in our daily lives, and they are frequently entrusted with making critical decisions that affect individuals and society. Image identification, recommendation algorithms, e-commerce, and online advertising have all profited from $\mathrm{AI}$ and machine learning (ML) applications over last decade. With the inclusion of AI and machine learning in such usages, there has been an increase in alarming reports of many types of biases, including gender, racism, and other factors, caused by these systems and the system. A very common question nowadays is, "What is bias?". Though bias can be defined in a number of ways, a common definition is - Algorithmic bias arises when a machine learning model generates outputs that are inherently skewed as a result of erroneous assumptions. Bias can enter the system at various stages, planning, collection of data, during the time of analyses of data, or while publishing a particular research work. As these systems become more commercialised, the negative impacts of bias are becoming severe and hence attracting attention by data scientists for solutions. The knowledge of data scientists regarding biases is increasing along with awareness of it, and hence they are working on mitigating the problem of bias.

\section{KEY WORDS: FAIRNESS AND BIAS IN AI/ML, ARTIFICIAL INTELLIGENCE, MACHINE LEARNING, BIAS}

\section{INTRODUCTION}

In the last 3 years, there has been a significant increase in overall use of AI across the globe. The percentage of enterprises using AI has gone up from 15\% to 37\%. Machine algorithms are increasingly being employed in high-stakes decisions such as loan applications, dating, and hiring. Algorithmic decision-making has significant advantages; unlike humans, robots are never bored or exhausted, and they can examine a wide range of considerations, far more than humans can. Algorithms, like humans, are prone to biases that cause them to make "unfair" conclusions (Althubaiti et al., 2016).

Unfair algorithm: An unfair algorithm is one that generates judgements that promote a specific group of people (Alexandra et al., 2016).

For example, RACIAL BIAS was anticipated by the COMPAS algorithm for forecasting re-offending risk,

Biosc Biotech Res Comm P-ISSN: 0974-6455 E-ISSN: 2321-4007

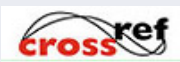

Identifiers and Pagination

Year: 2021 Vol: 14 No (9) Special Issue

Pages: 227-234

This is an open access article under Creative

Commons License Attribn 4.0 Intl (CC-BY).

DOI: $h t t p: / / d x . d o i . o r g / 10.21786 / b b r c / 14.9 .43$ which forecast larger risk values for black defendants than their actual risk. In another incident, it was discovered that Google's Adverts engine for targeted advertising served much fewer commercial for positions with high salaries to women than to men - GENDER BIAS.

As a consequence of these occurrences, public worry about the negative consequences of AI on our lives is developing. AI-assisted decision-making has the potential to amplify pre-existing prejudices while also introducing new classifications and criteria with new biases.

In this paper, we discuss what bias really means and its various types using some real-life examples. After understanding bias, we learn ways to mitigate it and account for it. We also analyse what "fairness" means and how can we ensure that our model is fair.

We categorise the works into three groups:

What is Bias?: Bias has a number of definitions and is generally treated as a negative term. Bias can enter through various aspects of AI/ML research, like, bias in data collection, bias due to under and over representation of population, analysis to get an already decided result, etc (Maria Šimundic 2013; Pham et al., 2019). 
Some of the common definitions of bias are-

- Any pattern or variation from the truth in data collection, analysis, interpretation, and dissemination that could lead to inaccurate conclusions is classified as bias.

- When the data available isn't representative of the population or issue being examined, it's called data bias

- Bias can generally mean systematic favouritism of a group over another.

- An AI system's decision that is prejudiced in favour of or against single human or set of persons, especially in an unjust manner, is referred to as bias.

Types of Bias: Bias can be classified into various types and its definition divided into different categories. We have discussed different bias below-

Cognitive Bias: Cognitive biases are intrinsic dispositions to comprehend and process information depending on our prior experiences, preferences, and in some cases, genetic wiring. There are 5 major types of cognitivebias-

Confirmation-Bias: It is said that the data will confess to almost anything if tortured long enough. When people deliberately seek out and favour information or data that supports their beliefs or preconceptions, they ignore or disregard contradictory or mitigating facts. It's a form of cognitive bias or purposeful bias. An intentional bias or explicit bias is when there is a deliberate tailoring of the data to fabricate the results of an experiment or study. This type of bias embroiders the information collected to move in a certain and predetermined direction to get a result which is required. In such biases, the study is not done to get results but rather a test is performed in a way to explain a presumed hypothesis (Zhang et al., 2018).

Participants in a confirmation bias study on the 2004 United State presidential election, for example, strong preconceived notions about candidates. The review concludes that pre-existing opinions about the candidates produced significant prejudice, resulting in erroneous judgement and illogical interpretation, after analysing contradictory utterances by the various contenders. This revealed that social reasoning is prone to confirmation bias (Dwork et al., 2011; Wadsworth et al., 2018).

Survivorship-Bias: The logical fallacy of focussing on the people or things that made it past some sort of selection process and neglecting those who did not, usually due to their lack of visibility, is known as survival bias. This can lead to erroneous conclusions in a variety of ways. Because failures are neglected, survivorship bias can lead to unduly optimistic beliefs. Some examples of survivorship bias (Christopher et al., 2010).

Is Your Friend Really Photogenic?: We all have friends whose social media feeds are loaded with incredible pictures, and we all believe they are photogenic. Is this actually the case? There's a good chance that pal isn't as flawless as the internet portrays. They could simply be spending a lot of time taking images in the hopes that at least one of them would be chosen. What we see is just one among many images, leading us to draw the incorrect idea that our friends are photogenic.

Is It True That Asians Thrive At Computer Science?: Asians are frequently thought to be skilled in computer technology and mathematics in the West, to the point where it has become a stereotype. Unfortunately, this isn't entirely correct. It's possible that some of it is merely a numbers game Despite the fact that just 10\% of Asians are computer and math experts, Asia's population is much larger than Americans. Humans present in the West may have had more exposure to those particular Asians, resulting in this widely held belief. This is a great example of concentrating entirely on the task at hand.

Sunk-Cash-Fallacy: The sunk cost fallacy refers to people's propensity to make judgments based on how much they've already committed, which leads to even greater investment but no rewards.

\section{Some Examples of Sunk Cash Fallacy-}

\section{- Breaking the diet and sunk costs}

Assume we were on a rigorous diet at the time, but we blew it at lunch when we indulged in a fantastic pizza. You intended to eat healthy for dinner, but since your diet has already been ruined, you might as well go all out and eat something terrible for supper as well. The sunk cost fallacy is illustrated here (Woodworth et al., 2017).

\section{- Advertising and the sunk cost fallacy}

Consider this scenario: you've spent a lot of money promoting a product, but sales aren't keeping up with the promotion. Unfortunately, it's all too easy to believe that because you've already spent so much, there's no point in stopping now and that you should go all out and spend even more money on marketing (Bruce \& Frey 2018) (Colin \& Blyth et al., 1972).

False-Causality-Bias: We are continually looking for patterns as data scientists. As a result of this proclivity, we occasionally discover patterns where none exist. When it is falsely assumed that if two events occur together, one must have caused the other.

An examples of false causality bias- In the 1980s, a professor looking into the causes of crime in New York City discovered a substantial link between the number of serious crimes and the amount of ice cream sold by street sellers. Should we, however, conclude that eating ice cream causes people to commit crimes? Because this makes little sense, we should assume that both were caused by an unseen variable. Summer is when crime rates are highest, and it is also when the majority of ice cream is sold. Ice cream sales aren't related to crime, and crime isn't the cause of sale of ice-creams. 
Availability Bias: The phrase "availability bias" describes how data scientists make assumptions based on realworld data or current knowledge. They believe data that is instantly available is relevant data. An example of availability bias-If someone says that drinking beer doesn't make you fat because Mr. A drinks beer everyday and is still not fat, this is an example of availability bias as the conclusion that beer doesn't make you fat is based on limited data which can just be an exception and this conclusion can be invalid (Cynthia 2012).

Types of Bias During Trial: Bias during trial can be of different types. There are three major stages of trial and each stage has different kinds of bias.

The three different stages are -

i. Pre-trial

ii. During trial

iii. After trial

Pre - trial bias

During this stage, there are 3 types of bias-

Flawed Study Design: Risks and outcomes of the study are stated ambiguously and with no objectives and validated methods. Standardization and blind are not taken into consideration (Eirini et al., 2019; Kamiran \&t Calders 2012).

Selection Bias: When the participation pool, or subsequent data, is not representative of the targeted respondents, a selection bias occurs.

Channeling Bias: When a medicine is preferentially administered to patients with differing baseline characteristics, this is known as channelling bias

\section{Bias During Trial}

Interviewer Bias: It is the effect of the interviewer's characteristics and the types of questions they ask. Personal opinions and tendencies of an interviewer can lead to prejudice.

Chronology Bias: When earlier recruited study participants allocated into one intervention group, and later participants ending up in another group.

Recall Bias: Participants in studies may occasionally provide incorrect responses that rely on their capacity to recall earlier events. As a result of recall mistake, the prejudice in this scenario is referred to as recall bias.

Transfer Bias: When study cohorts experience unequal losses to follow-up, transfer bias might arise .

Exposure Misclassification: It's a type of cognitive bias. A systematic error known as misclassification bias might arise at any point during the research process. It happens when a person is placed in a category to which they are not supposed to belong.

Performance Bias: Performance bias occurs when one group of subjects in an experiment receives more attention from investigators than another. There are systematic disparities between groups as a result of the variance in treatment levels, making true results difficult or impossible to achieve.

\section{Bias After Trial}

The 2 types of bias in this step are-

Citation Bias: The propensity for positive study findings to be quoted more frequently than neutral or negative findings.

Confounding Bias:When two or more factors are individually related with both the desired outcome and the exposure, confounding occurs.

\section{Some Other Types of Biases}

Historical Bias: the prejudice and the social and technical concerns that already exist around us and can permeate into the process of data generation, even with ideal sampling and/or feature selection. As a consequence of the fact that only 5\% of 500 CEOs are female, searching for female CEOs yielded fewer female CEO images in a picture search result from 2018, skewing the search results in favour of male CEOs. Of course, these search results reflected reality, but the notion of whether or not search engines should mirror actuality is worthy of discussion. Measurement Bias is caused by the way we select, use, and measure a specific trait.

Prior arrests and arrests of friends or family members were utilised as proxy variables in the recidivism risk prediction tool COMPAS to forecast the amount of "risk profile" or "criminal behaviour," which could be considered mismeasured proxies on their own. This indicates towards the fact that minority groups are more frequently inspected and kept tabs on, resulting in greater arrest rates. However, people from minority groups should not be assumed to be more dangerous simply because they have a greater arrest rate. There are differences in how these groups are assessed and handled (Kamiran \&t Calders 2012).

Aggregation Bias: arises when incorrect inferences about a subgroup are generated based on data from other subgroups, or when faulty beliefs about a community have an impact on the model's output and definition in general. This type of bias is noticed in clinical aid techniques and tools. Consider a set of diabetic people who appear to differ by nationality and gender, or $\mathrm{HbA1c}$ readings, which are regularly used in diabetes diagnosis and monitoring and differ in a variety of ways across genders and nationalities. Because of all of these characteristics, as well as their varying meanings and impacts among sub-classes and populations, a general prototype is unlikely to be the best fit for all of us. According to a study on Machine Learning Bias and Fairness, population is categorized into five different 
groups. This is true even if they are equally represented in the training data (Farnadi et al., 2018).

Population bias occurs when the user audience represented in the dataset or platform differs from the target group in terms of statistics, demographics, representatives, and user attributes. Men are more likely to use online forums such as Reddit or Twitter, while women are thought to prefer Pinterest, Facebook, and Instagram.

The Simpson's Paradox skews data that is made up of subgroups or individuals who behave differently. According to Simpson's paradox, a trend, association, or characteristic identified in underlying subgroups can differ substantially from the relationship or characteristic detected when these subgroups are aggregated. When our judgement is influenced by the behaviour or content of others, this is known as social bias. When we are attempting to appraise or analyse an item that has a low rating but is persuaded by other high ratings, we alter our score, believing that we are being too harsh.

Real-Life Examples of Bias: In this section, we examine several real-world examples of bias in AI and machine learning to gain a better understanding of what bias is and the repercussions of allowing it to exist.

Racism Embedded In US Healthcare: In October 2019, researchers uncovered that an algorithm used in US hospitals to predict which patients would require more medical care preferred white patients over black patients While race was not a factor in this investigation, healthcare spending history was found to be highly associated to race. The concept was that a person's healthcare needs might be summed up by their cost. Black patients with the same ailments had lower healthcare costs on average than white patients with the same conditions for a variety of reasons (Hennekens \& Buring 1987).

Solution: Because racial health disparities are driven by a multitude of causes, many of which act in concert, therapies must take into account all of the ways racism influences health care. Interventions must focus on physicians, patients, and their interactions because both physicians and patients play a role in the success of medical care. Other biases are deeply embedded in the health-care system, needing structural reforms. Racial disparities in health care can be decreased by enacting policies that address the many factors that contribute to racism (Louis et al., 2015).

Amazon's Hiring Algorithm: Amazon is one of the world's most powerful technology companies. As a result, it's no wonder that machine learning and artificial intelligence are heavily used by them. Amazon discovered in 2015 that their algorithm for hiring staff was skewed against women. The reason for this was that the algorithm had been programmed to favour males over women based on the number of resumes submitted over the preceding ten years and the fact that men made up the majority of the candidates (Zafar et al., 2017).
2016 Us Elections: During the 2016 presidential election, many people thought Hillary Clinton and Donald Trump were untrustworthy and unlikable; in fact, just a third of voters said either candidate was "honest and trustworthy." Despite this, Donald Trump appears to be the campaign's most unpopular candidate. Clinton received more favourable coverage in six major news outlets, according to Data Face. (Including the New York Times, Wall Street Journal, Chicago Tribune, and Washington Post). Donald Trump was declared the winner, despite forecasts that Hillary Clinton would be the next president. Desirability in social situations the gap could be due to bias (Goodfellow et al., 2014).

Social Desirability Bias: This phenomenon occurs when some respondents explain their response embroidered to make it more socially acceptable rather than their "true" reaction (Harini et al., 2019).

Facebook's Ad's Algorithm: After allowing advertisers to target ads based on gender, ethnicity, and religion, all of which are legally protected categories in the US, Facebook was found to be in violation of the US constitution in 2019. Nursing and secretarial jobs primarily targeted women, whereas janitors and taxi drivers primarily targeted men, particularly those from minority backgrounds. Because the algorithm determined that providing real estate ads to white people resulted in higher engagement rates, minorities were no longer seen the adverts. This issue develops as a result of the learning process of the AI computer. As is the nature of machine learning algorithms, the ad platform developed a pattern from the data it was given, but the pattern represented existing societal imbalances and, if left unchecked, would have contributed to perpetuate them further.

Beauty Pageant Algorithm Prove To Be Racist: Thousands of people participated from all across the world and submitted their photographs to be judged by robots in an international beauty competition in 2016. Beauty. To determine what idea of "true beauty" is held by the computer, AI was meant to employ an advanced algorithm without the involvement of human bias. However, things quickly went wrong when the algorithm began to associate skin colour with attractiveness, selecting winners only on the basis of race (Matt et al., 2017).

Mitigation of the Bias: We now have a deep understanding of what bias is and its various forms after reading about the different types and analysing some real-life examples. After understanding bias, we now see how it can be ameliorated. We also discuss the legal issues of mitigating bias. There are three different approaches of mitigating bias from the process of machine learning-

Pre-Processing: Because the data source and extraction procedures may introduce unwanted bias, effective bias mitigation begins from the data collection and processing phase. As a result, the greatest amount of work should be spent into evaluating the data source's integrity and 
ensuring that the data collection procedure incorporates adequate and trustworthy measuring methodologies. The majority of data was collected through questionnaires prior to the "big data" era. This enabled for the creation of experimental designs that used statistical analysis to control for potential biases. Today's technology allows us to access vast volumes of data at little cost, but knowledge on the circumstances in which the data was collected is typically scarce (Walker 2014; Hardt 2017; Srebro et al., 2017).

As a result, pre-processing procedures make sure that the data fed as input is fair and equitable. Hide the protected attributes, change the data set's class labels, and reweight or resample the data are all options. To guarantee that the data sample is representative, it may be essential to rebuild omitted or censored data in some cases. There are several imputation approaches that may be used to accomplish this goal, with hot deck operations being one of the more effective (Mehrabi et al., 2019; Paul 2011).

In-Processing: The in-processing algorithms are the second sort of mitigation strategy. In this circumstance, unintentional bias is prevented directly during the training period. Incorporating a fairness penalty directly into the loss function is a simple method to achieve this goal. A decision boundary covariance limitation is one strategy for logistic regression or linear SVM. A meta-algorithm, on the other hand, accepts an input of a fairness metric and outputs a new classifier that is optimal for that metric. Debiasing-based adversarial classification was also made possible by the invention of generative adversarial networks (GANs). In this discipline, the capacity of an adversarial neural network to predict a protected property is minimised, while a neural network classifier is taught as a typical predictor.

Post-Processing: A post-processing method is used in the third category of mitigation algorithms. Only the output of a trained classifier is changed in this case. The Bayes optimum equalised odds predictor can be used to adjust the output labels in order to accomplish an equivalence aim. A paper with different approach employs soft classification based on proxy model outputs to offer a weighted estimate for demographic disparity. Post-processing methods have the advantage of providing fair classifiers without requiring the original model to be retrained, which possibly is time intensive as well as difficult to use in production. This strategy, on the other hand, may reduce accuracy or jeopardise any generalisation gained by the initial classifier.

Accounting for Bias: The assignment of responsibility for how an algorithm is produced and its impact on society is referred to as algorithmic accountability. The problem is worsened in AI systems since the solution is inferred rather than specified utilising machine learning techniques and complex data. The most essential are discussed below, and they account for bias either proactively, through bias-aware data collection, or retroactively, by explaining AI conclusions in human terms; we also stress the importance of recognizing and recording prejudice through formalizations such as ontologies.

Proactively: To address a variety of demands, a variety of data collection methods are employed; nevertheless, these methods may create bias throughout the data collection stage. Individual bias checklists and elicitation tasks, as well as mathematical pooling, group elicitation and consensus building, and hybrid bias elicitation in evidence synthesis methodologies, have all been provided for a systematic approach to bias elicitation in evidence synthesis. Bias elicitations have been demonstrated to be biased even when high-quality assessors are utilised, and treatments have been suggested. To deal with data and label shortages, crowdsourcing is a typical machine learning approach that relies on large-scale human input. Because people from varied ideological and cultural backgrounds are involved, biased labelling may be visible to all of them, making it more difficult to minimise biases in crowdsourced data.

Describing And Modelling Bias Using Ontologies: Not only does bias analysis necessitate a thorough comprehension of the many sources, but it also necessitates the interpretation and description of bias's meaning, potential side effects, provenance, and context. Unbalanced categories are sometimes misconstrued as prejudice and seen as potential sources of unfavourable results. Furthermore, knowledge bases and ontologies, like any other data source, are prone to bias and imbalance in knowledge. For instance, the amount of research done on disorders linked to these genes may change descriptions of existing gene mutations in a knowledge base like COSMIC or the Gene Ontology properties associated with a gene.

Expressive formal prototypes are necessary to capture and validate the properties of a source of data, as well as the conditions or environment in which the data source is skewed. Bias can be measured and detected in a wide range of data sets using formalizations like description and causal logics. They also make it possible to annotate claims with trustworthiness, timing, and causal linkages. Ontologies can employ classes, properties, and subsumption linkages, as well as contextual representations of these notions, to build a universe of discourses. Existing logic-based and ontological formalisms aren't meant to capture contextual bias or identify imbalanced categories that frequently correlate to real-world events, despite their expressiveness.

As a result, expressive ontological formalisms are required to characterise the contextual characteristics of various types of sources, such as data sets, knowledge bases, and ontologies, as well as annotations demonstrating the causality and provenance of the represented knowledge. These formalisms will provide reasoning processes to bias detection algorithms, allowing them to enhance accuracy while clarifying the meaning, conditions, source, and context of prejudice. As a result, context-aware bias description and interpretability will be possible thanks to domain modelling with ontologies. 
Retroactively: The ability to explain the internal mechanics of a learning model in human words is known as explain ability. It's sometimes confused with interpretability, but the latter refers to the model's ability to predict what will happen if the inputs or parameters are changed. Although there have been attempts to address interpretable ML for some time (Hoffman $\mathrm{Ct}$ Klein, 2017), the academic literature has expanded in recent years, with terminology such as explainable AI (Adadi \& Berrada, 2018) and black box explanation (Adadi \& Berrada, 2018) gaining popularity. Guidotti and his colleagues are a group of people that are passionate about what they do (Guidotti et al., 2019).

To decipher a model's global logic, many studies propose building an interpretable classifier that can imitate the perplexing decision mechanism. (Montavon et al., 2018) used these methodologies to explain specific models like deep neural networks. Only a few people dispute the black box paradigm (Henelius et al., 2014). Transparent classifiers that are self-interpreting and have predicted accuracy comparable to cryptic techniques have been created due to the difficulty of ex post characterising black boxes and sophisticated models. Bayesian models (Li \& Huan, 2017), generalised additive models (Lou et al., 2013), supersparse linear models (Ustun \& Rudin, 2016), rule-based decision sets (Lakkarajuet al., 2016), optimal classification trees (Bertsimas \& Dunn, 2017), model trees (Broelemann \&t Kasneci, 2017), model (Zhang et al., 2018).

Fairness: There is no standard definition of fairness that is used world-wide but there are more than 20 different definitions. Some of the definitions are listed below-

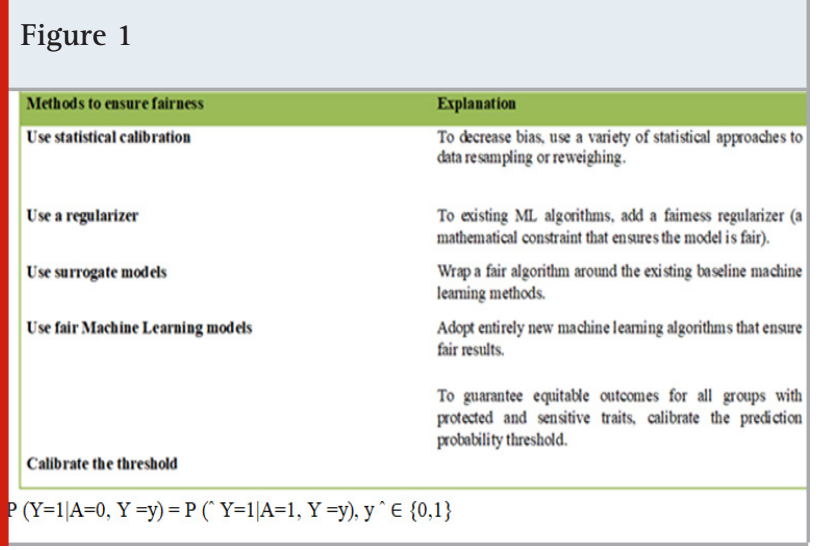

Equalized Odds: The concept was first defined in the context of binary classes. Blake Woodworth expanded the concept for a number of sessions in 2017 . Equalised odds, also known as conditional procedure accuracy equality and disparate treatments in machine learning, is a fairness metric. If the true positive and false positive rates in the protected and unprotected groups are the same, the classifier meets this condition satisfying the formula:

Demographic-Parity: According to demographic parity, all members of a protected group (such as gender) should have an equal chance of success. The favoured decision is one that results in a positive outcome, such as "getting into university," "receiving a loan," or "being seen the ad." The difference should ideally be zero, as previously indicated, however this is not always the case.

Fairness Through Awareness: When an algorithm makes similar predictions for similar people, it is said to be fair. To put it another way, any two people who are comparable in terms of a task-specific similarity (inverse distance) metric should provide the same results.

Treatment Equality: When the ratio of false negatives to false positives for both protected group categories is the same, treatment equality is attained.

Test Fairness: According to the test fairness requirement, participants in two groups - protected and unprotected (female and male) - must have an equal chance of accurately belonging to the positive class for any projected probability score S.

Counterfactual Fairness: According to the notion of counterfactual fairness, a choice is fair to an individual if it is the same in the real world and a hypothetical world in which the individual belongs to a different demographic group.

Fairness in Relational Domains: A fairness concept capable of capturing the relationship structure of a domain-not just by looking at individual attributes, but also by looking at social, organisational, and other links between people.

Conditional Statistical Parity: Given a collection of acceptable considerations, what should you do? Participants in both the protected and unprotected (female and male) groups should have an equal chance of reaching a favourable outcome, according to conditional statistical parity (L).

Future Directions and Conclusions: There are several unturned stones in this field and still a huge scope of growth. Firstly, despite the fact that there exists a number of bias mitigation techniques but there are no conclusive results as to how efficient the method of mitigation is or on what basis should the mitigation method be selected or which method is best for which type of bias. As there are so many unanswered questions, the problem of bias cannot be fully resolved. There is a need for a comprehensive assessment of existing approaches in order to gain a thorough understanding of their efficacy and limitations, as well as to give more options. The problem with having a system is that different methods perform differently with different bias.

This issue must be solved by using a standard data set. Therefore, an internationally accepted evaluation must be developed which is used to check the performance efficiency of the model and also covers all the fairnessrelated aspects. Secondly, the definition of "fairness" is a problem. There is no standard definition of fairness, 
but multiple definitions occur which make it difficult to understand what fair is as "fair is not fair everywhere". Therefore, a standard definition of "fairness" must be derived to making judging a model as fair or unfair easier.

Thirdly, the data scientists and all the other members involved in ML must be aware of all types of bias and the concept to fairness. Regular checks on the ML multidimensionally will ensure a fair model. Two strategies could be used as a solution for addressing the problem of bias:

\section{CONCLUSION}

To summarise, the issue of bias and unfairness in AI/MLbased decision-making systems has recently attracted a lot of attention. This research looks at some real-life examples of prejudice and how to overcome it to help readers understand what it is. We also talk about the issue of a nebulous sense of fairness. Finally, we'd like to emphasise that prejudices are profoundly ingrained in our cultures, and it's a misconception to expect that the AI and prejudice issues can be solved solely by technical means. Because technology reflects and projects our biases into the future, it is the responsibility of technology developers to recognise the limitations of the technology and implement safeguards to avoid any issues.

\section{REFERENCES}

Alaa Althubaiti, 2016, "Information bias in health research: definition, pitfalls, and adjustment methods", Journal of Multidisciplinary Healthcare.

Alexandra Olteanu, Carlos Castillo, Fernando Diaz, and Emre Kiciman, 2016, "Social data: Biases, methodological pitfalls, and ethical boundaries”, Microsoft Research. Analytics India, 2017, "8 Real Life Examples When Algorithms Turned Rogue, Causing Disastrous Results”, Analytics India magazine.

Ana-Maria Šimundi囚, 2013, "Bias in research”, University Department of Chemistry, University Hospital Center "Sestre Milosrdnice”, Zagreb, Croatia.

Anh Pham, Michael Cummings, Cliff Lindeman, Neil Drummond, Tyler Williamson, 2019, "Recognizing misclassification bias in research and medical practice", Family Practice, Volume 36.

B. H. Zhang, B. Lemoine and M. Mitchell, 2018, "Mitigating Unwanted Biases with Adversarial Learning," Association for the Advancement of Artificial Intelligence.

Blake Woodworth, Suriya Gunasekar, Mesrob I. Ohannessian, Nathan Srebro, 2017, "Learning NonDiscriminatory Predictors”, Cornell University.

Bruce B. Frey, 2018, “Interviewer Bias”, The SAGE Encyclopaedia of Educational Research, Measurement, and Evaluation.

Bryn Farnsworth, PostDoc. \& Expert Content Writer, 2020, Imotions.
C. Dwork, M. Hardt, T. Pitassi, O. Reingold and R. Zemel, 2011, "Fairness Through Awareness".

C. Wadsworth, F. Vera and C. Piech, 2018, "Achieving Fairness through Adversarial Learning: An Application to Recidivism Prediction," CoRR.

Christopher J. Pannucci and Edwin G. Wilkins, 2010, "Identifying and Avoiding Bias in Research", Plastic and Reconstructive Surgery.

Colin R Blyth, 1972, On Simpson's paradox and the sure-thing principle. J. Amer. Statist. Assoc. 67, 338 (1972), 364-366.

Cynthia Dwork, Moritz Hardt, Toniann Pitassi, Omer Reingold, and Richard Zemel, 2012, "Fairness through awareness", Innovations in Theoretical Computer Science.

Drew Westen, Pavel S. Blagov, Keith Harenski, Clint Kilts, and Stephan Hamann, 2014, "Neural Bases of Motivated Reasoning - An fMRI Study of Emotional Constraints on Partisan Political Judgment in the 2004 U.S. Presidential Election”, Data science association. Eirini Ntoutsi, Pavlos Fafalios, Ujwal Gadiraju, Vasileios Iosifidis and Wolfgang Nejdl, 2019, "Bias in data-driven artificial intelligence systems-An introductory survey", L3S Research Centre \& Faculty of Electrical Engineering and Computer Science, Leibniz University Hannover, Hannover, Germany.

F. Kamiran and T. Calders, 2012, "Data preprocessing techniques for classification without discrimination," Knowledge and Information Systems.

Faisal Kamiran and Toon Calders, 2012, "Data preprocessing techniques for classification without discrimination”, Knowledge and Information Systems. FlyData, 2020, “Cognitive Biases in Data Science”, Data engineering.

Golnoosh Farnadi, Behrouz Babaki, and Lise Getoor, 2018, Fairness in Relational Domains, AIES.

Goodfellow, J. Pouget-Abadie, M. Mirza, B. Xu, D. Warde-Farley, S. Ozair, A. Courville and Y. Bengio, 2014, "Generative Adversarial Nets", in Advances in Neural Information Processing Systems.

Harini Suresh and John V Guttag, 2019, “A Framework for Understanding UnintendedConsequences of Machine Learning”, Cornell University.

Hennekens CH, Buring JE, 1987, "Epidemiology in Medicin”, Little, Brown, and Company, Boston.

Jack Beck and Nick Sorscher, 2016, "Trump and the Media: A Text Analysis”, The Data Face.

Logically, 2019, "5 Examples of Biased Artificial Intelligence".

Louis A. Penner, Irene V. Blair, Terrance L. Albrecht and John F. Dovidio, 2015, "Reducing Racial Health Care Disparities: A Social Psychological Analysis”, The National Center for Biotechnology Information.

M. B. Zafar, I. Valera, M. G. Rodriguez and K. P. Gummadi, 2017, "Fairness Constraints: Mechanisms for Fair Classification", in Proceedings of the 20th International Conference on Artificial Intelligence and 
Statistics.

Matt J Kusner, Joshua Loftus, Chris Russell, and Ricardo Silva, 2017, “Counterfactual Fairness”, NIPS.

Michael Walker, 2014, "The Deadly Data Science Sin of Confirmation Bias”, Data Science Central.

Moritz Hardt, 2017, "Intro to observational fairness criteria”, University of California.

Moritz Hardt, Eric Price, Eric Price, Nati Srebro, 2016, "Equality of Opportunity in Supervised Learning", NeurIPS.

Ninareh Mehrabi, Fred Morstatter, Nripsuta Saxena, Kristina Lerman, Aram Galstyan, 2019, “A Survey on Bias and Fairness in Machine Learning “, Cornell University.

Paul de Vries, 2011, "Equal Opportunity”, Blackwell Reference.

Paul J. Lavrakas, 2008, "Social Desirability", Encyclopaedia of Survey Research Methods.

Petri H, Urquhart J, 1991, "Channeling bias in the interpretation of drug effects”, Stat Med.

Prabhakar Krishnamurthy, 2019, "Understanding Data Bias”, Towards Data Science.

Priya Dialani, 2020, "Top 5 Biases in Data Science to know for model's accuracy”, Analytics Insights.

R. R. Andridge and R. J. Little, 2010, "A Review of Hot Deck Imputation for Survey Non-response," International Statistical Review.

Rahul Aggarwal, 2020, "Five Cognitive Biases in Data Science (And how to avoid them)", Towards Data Science.

Ricardo Baeza-Yates, 2018, "Bias on the Web”, Commun. ACM 61.

Richard Berk, Hoda Heidari, Shahin Jabbari, Michael Kearns, and Aaron Roth, 2017, "Fairness in Criminal Justice Risk Assessments: The State of the Art”, Research
Gate.

S. Barocas, M. Hardt and A. Narayanan, 2018, "Limitations and Opportunities", Rev. research and advanced ML.

S. Venkatasubramanian, A. Friedler and C. Scheidegger, 2016, "On the (im)possibility of fairness," CoRR, vol. abs/1609.07236.

Sahil Verma and Julia Rubin, 2018, IEEE.

Samara Klar, Christopher R. Weber and Yanna Krupnikov, 2017, "Social Desirability Bias in the 2016 Presidential Election”, De Gruyter.

Sray Agarwal, Rashed Haq, Rodney Coutinho, 2019, "Fair AI: How to Detect and Remove Bias from Financial Services AI Models”, Publicis Sapient.

Stephanie Glen, 2021, "Welcome to Statistics How to", Statistics how to.

T. Adel and A. Weller, 2019, "One-network Adversarial Fairness," Aaai.

Tamm M, Hilgers RD, 2014," Chronological Bias”, Methods Inf Med.

Terence Shin, 2020, "Real-life Examples of Discriminating Artificial Intelligence”, Towards Data Science.

Ting Wang and Dashun Wang, 2014, "Why Amazon's ratings might mislead you: The story of herding effects", Big data 2, 4 (2014), 196-204.

Valeria Cortez, 2019, "How to define fairness to detect and prevent discriminatory outcomes in Machine Learning”, Towards Data Science.

Value Tortoise, 2021, "12 real life examples of survivorship bias you may not have known", Value Tortoise.

Wikipedia - Survivorship Bias.

Will Goodrum, 2017, "Statistical \& Cognitive Biases in Data Science: What is Bias?”, Elder Research. 